\title{
Phase or Amplitude? The Relationship between Ongoing and Evoked Neural Activity
}

\author{
Chencan Qian (钱晨灿) $)^{1,2}$ and Xin Di (邸新 $)^{3}$ \\ ${ }^{1}$ State Key Laboratory of Brain and Cognitive Science, Institute of Biophysics, Chinese Academy of Sciences, Chaoyang District, Beijing 100101, China, \\ ${ }^{2}$ Graduate University of Chinese Academy of Sciences, Beijing 100101, China, and ${ }^{3}$ Department of Radiology, University of Medicine and Dentistry of New \\ Jersey-New Jersey Medical School, Newark, New Jersey 07103 \\ Review of Scheeringa et al.
}

Whether performing a task or resting, our brains exhibit continuous neural activity, which at any instant comprises the evoked response to a given task and ongoing activity. There is increased interest in this ongoing activity because accumulating evidence has shown that it can influence perception, cognitive control, and motor performance (Coste et al., 2011). It is currently hypothesized that ongoing activity reflects internal models of the environment that, among other things, afford adaptation and prediction (Berkes et al., 2011). In the domain of perception, both prestimulus alpha band power (van Dijk et al., 2008) and phase (Mathewson et al., 2009) have been shown to predict whether a near-threshold stimulus can enter conscious awareness. On a much coarser temporal scale, prestimulus neural activity in fusiform face area, as measured by functional magnetic resonance imaging (fMRI), can bias an ambiguous Rubin's vase-faces picture to be perceived as a face (Hesselmann et al., 2008).

To understand how ongoing activity influences perception, unraveling the re-

Received May 2, 2011; revised May 25, 2011; accepted May 27, 2011.

This work was supported by grants 30921064 and 90820307 from the National Natural Science Foundation of China (NSFC) to C.Q. and grants 1R01AG032088-01A2 and 5R01NS049176-04 from the NIH to X.D. We wish to thank Drs. Zuxiang Liu, Bharat Biswal, Glyn Humphreys, Huan Luo, and Ji Chen for very helpful comments and improvement of English on an earlier version of this manuscript.

Correspondence should be addressed to Dr. Xin Di, ADMC Building. 5, Suite 575, 30 Bergen Street, Newark, NJ 07103. E-mail: synge.x.d@gmail.com.

DOl:10.1523/JNEUROSCI.2197-11.2011

Copyright $\odot 2011$ the authors $\quad 0270-6474 / 11 / 3110425-02 \$ 15.00 / 0$ lationship between ongoing and evoked activity is essential. However, it is still not clear how the evoked activity is controlled by the amplitude or phase of ongoing activity. One methodological challenge is how to unambiguously distinguish between ongoing and evoked activity in the observed neural activity. Scheeringa et al. (2011b) simultaneously recorded activity with electroencephalography (EEG) and fMRI as subjects performed a visual task. This experimental design made it possible to identify ongoing and evoked activity with different methods, for example, measuring ongoing activity with EEG and evoked activity with $\mathrm{fMRI}$.

A wedge-shaped stimulus was briefly presented in subjects' lower-left visual field for $17 \mathrm{~ms}$. Subjects were asked to respond to a color change in a central fixation point, which was unrelated to the peripheral stimulus. Independent component analysis was applied to the EEG data to remove noise and identify a single component with a clear alpha band peak and a topography suggesting that it was derived from the visual cortex. The alpha band amplitude and phase of the component were calculated to classify the evoked fMRI responses.

The result revealed that the power and phase of ongoing activity are functionally dissociated in modulating evoked activity. First, the event-related BOLD responses were sorted according to higher or lower alpha band amplitudes at stimulus onset time. For this comparison, baseline activity needed to be controlled because previous studies had shown that alpha amplitude in visual cortex is negatively correlated with the BOLD signal. Indeed, although the trials following higher alpha amplitudes had smaller activity in the visual cortex, after removing the baseline activity from the pseudo-trials in which no stimulus was delivered, the trials following higher and lower alpha amplitude tended to evoke similar net activity. However, when the event-related BOLD responses were sorted by the alpha phase, the trials presented at the trough of the alpha cycle induced larger BOLD responses than trials presented at the peak. These effects were mainly in Brodmann's area 17 and were weaker in Brodmann's areas 18 and 19.

Scheeringa et al. (2011b) demonstrated a seminal way of combining the complementary temporal and spatial information provided by electrophysiological and hemodynamic methods. Previous studies had probed both ongoing and evoked activity with either EEG or fMRI. However, for EEG it is not easy to localize the measured activity, which might be vital for explaining its cognitive consequence, whereas for $\mathrm{fMRI}$ it is not possible to access the phase of ongoing neural oscillation in a given frequency band. One idea of combining the two methods is to apply them simultaneously to a single neural process of interest, e.g., the evoked activity, guiding EEG sourcing with fMRI results. Instead, Scheeringa et al. (2011b) used simultaneous EEG 
and fMRI to measure ongoing activity and evoked activity respectively, emphasizing different aspects of these two neural processes whose relationship was of concern. In addition, although the BOLD signal has been shown to correlate with EEG oscillation power (Scheeringa et al., 2011a), measuring ongoing and evoked activity with different imaging methods helps to attenuate any potential artifact that might arise from the data-sorting procedure, given that prestimulus and poststimulus activity are not independent. As noted by the authors, the limited localization accuracy of the ongoing oscillations and the temporal resolution of the evoked responses still require careful interpretation. Nevertheless, the article established for the first time the direct link between the fine temporal structure of ongoing neural oscillations and evoked hemodynamic responses, providing a neural correlation with relatively high spatial resolution for the cognitive impact of the fast fluctuating ongoing activity.

One remaining question is how to interpret the occipital alpha phase at stimulus onset. The ongoing occipital oscillations presumably arise from early visual cortices. However, it takes some time before the information about a new stimulus actually arrives at these cortical sites. Previous electrophysiological studies in monkeys (Schmolesky et al., 1998) suggest that the onset latency of visually evoked single-unit responses in V1 ranges from 34 to $97 \mathrm{~ms}$. In humans, the suppressive effect of transcranial magnetic stimulation over occipital cortices follows a U-shaped function maximized between 80 and $130 \mathrm{~ms}$ after stimulus onset (Kammer, 2007). As a consequence, stimuli that appear at the optimal phase are not necessarily processed in the optimal phase. Nevertheless, larger evoked responses and better detection performance (Mathewson et al., 2009) for these items are indeed observed. This raises the possibility that the alpha phase at stimulus onset might actually reflect the phase one cycle (about $100 \mathrm{~ms}$ ) later in the same location, within the critical time window for information processing in occipital cortices. This hypothesis is consistent with the observation that the effect of phase was only reliable for high alpha power trials (Mathewson et al., 2009). Lower power suggests less synchrony and thus faster dephasing of the underlying neuron population, in which case the phase at stimulus onset might not be a good indicator of the phase $100 \mathrm{~ms}$ later. However, after stimulus onset the ongoing oscillations gradually become mixed with the evoked responses, and there can be significant phase resetting. Thus, the concept of ongoing os- cillations after stimulus onset remains to be further justified, and the question of what to infer about the effects of processing-time phase based on the onset-time phase is not trivial.

At first glance, the result that trials with different prestimulus alpha power induce similar net BOLD responses seems incompatible with previous findings that prestimulus alpha power predicts perception and performance. The resolution of this apparent discrepancy lies in the answer to the following two questions: is it the net or total neural response that correlates with perception, and does the feedforward or the feedback component contribute more to the observed BOLD signal? Although the prestimulus alpha power manifested no significant effect on the net evoked response, it did show an intrinsic negative correlation to the poststimulus baseline activity and thus modulated the total evoked response. This result suggests that perception and awareness might correlate with total rather than net neural response. Another notable implication is that although prestimulus ongoing phase and power might both influence stimulus processing, it is likely that there are different mechanisms, as phase directly modulates net evoked response while power only changes the baseline activity. The slow hemodynamic signal might also contain feedback ingredients, making the evoked BOLD response a result and not just a cause of perception. Feedback components can vary dramatically especially for near-threshold stimuli, as they elicit totally different percepts. However, the peripheral stimuli adopted in Scheeringa et al. (2011b) were well above threshold, in which case the feedback modulation effect should be largely reduced because of the more-orless similar perception leading to similar net BOLD responses. One significant benefit of using above-threshold stimuli is that they can control top-down factors, so that any difference observed in the net BOLD responses should be attributed more to a feedforward mechanism.

Considering the significant phase effect, it is also possible that pooling across different phases may have blurred the potential scaling effect of alpha power on event-related BOLD responses. Phase is thought to reflect the oscillating state of the neuronal ensemble, with troughs and peaks indicating excitability that is greater or less than the average, respectively. The amplitude or, equivalently, the power of this oscillation might thus act as a scaling factor of the foregoing modulation; e.g., the troughs with higher power might signify a stronger upregulation of the excit- ability of the population. However, when troughs and peaks are mixed together a near average excitability always results no matter whether the overall power is high or low. Consequently, analyzing the power effect for trials with troughs or peak phases separately may provide further insight into this issue.

Last but not least, the explanation of the results to date assumed that the ongoing activity and evoked activity were additive. However, it may not be the case under the framework of phase resetting (Makeig et al., 2002). The phase-resetting theory proposed that the amplitude of ongoing oscillations do not change after stimulus onset; in contrast, the phase of the oscillations becomes aligned to the stimulus onset. Whether the phase-resetting process is controlled by alpha oscillations remains unclear.

\section{References}

Berkes P, Orbán G, Lengyel M, Fiser J (2011) Spontaneous cortical activity reveals hallmarks of an optimal internal model of the environment. Science 331:83-87.

Coste CP, Sadaghiani S, Friston KJ, Kleinschmidt A (2011) Ongoing brain activity fluctuations directly account for intertrial and indirectly for intersubject variability in Stroop task performance. Cereb Cortex. Advance online publication. Retrieved April 6, 2011. doi:10.1093/cercor/bhr050.

Hesselmann G, Kell CA, Eger E, Kleinschmidt A (2008) Spontaneous local variations in ongoing neural activity bias perceptual decisions. Proc Natl Acad Sci U S A 105:10984-10989.

Kammer T (2007) Masking visual stimuli by transcranial magnetic stimulation. Psychol Res 71:659-666.

Makeig S, Westerfield M, Jung TP, Enghoff S, Townsend J, Courchesne E, Sejnowski TJ (2002) Dynamic brain sources of visual evoked responses. Science 295:690-694.

Mathewson KE, Gratton G, Fabiani M, Beck DM, Ro T (2009) To see or not to see: prestimulus alpha phase predicts visual awareness. J Neurosci 29:2725-2732.

Scheeringa R, Fries P, Petersson KM, Oostenveld R, Grothe I, Norris DG, Hagoort P, Bastiaansen MC (2011a) Neuronal dynamics underlying high- and low-frequency EEG oscillations contribute independently to the human BOLD signal. Neuron 69:572-583.

Scheeringa R, Mazaheri A, Bojak I, Norris DG, Kleinschmidt A (2011b) Modulation of visually evoked cortical FMRI responses by phase of ongoing occipital alpha oscillations. J Neurosci 31:3813-3820.

Schmolesky MT, Wang Y, Hanes DP, Thompson KG, Leutgeb S, Schall JD, Leventhal AG (1998) Signal timing across the macaque visual system. J Neurophysiol 79:3272-3278.

van Dijk H, Schoffelen JM, Oostenveld R, Jensen O (2008) Prestimulus oscillatory activity in the alpha band predicts visual discrimination ability. J Neurosci 28:1816-1823. 